\title{
Fit für die digitale Zukunft
}

\author{
M. Strugl
}

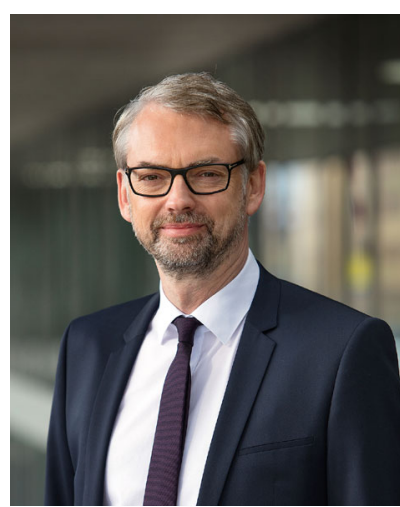

\section{LH-Stv. Dr. Michael Strugl, MBA}

Die Digitalisierung verändert unsere Gesellschaft. Internet der Dinge, Industrie 4.0, Blended Learning, Virtuelle Realität, Intelligentes Stromnetz, Ambient Assisted Living - die Bandbreite an Themen und Herausforderungen ist groß. Vor diesem Hintergrund hat sich das Land Oberösterreich entschlossen, mit einer Leitinitiative Digitalisierung dafür zu sorgen, dass unser Bundesland auch in einer digitalisierten Welt wettbewerbsfähig bleibt. Die Leitinitiative steht für das Bestreben Oberösterreichs, den Strukturwandel proaktiv zu begleiten und Potenziale für künftiges Wachstum zu erschließen. Zwei wesentliche Faktoren dafür - Künstliche Intelligenz als vielversprechende Zukunftstechnologie und potenzielles Alleinstellungsmerkmal Oberösterreichs sowie das Thema Fachkräfte - möchte ich in diesem Artikel näher beleuchten. Denn die Digitalisierung ermöglicht völlig neue Geschäftsmodelle und bringt die Chance auf mehr Beschäftigung. Gleichzeitig muss uns aber auch bewusst sein, dass auf der anderen Seite etablierte Geschäftsmodelle plötzlich zusammenbrechen, erworbenes Wissen überholt und Technologien veraltet sein können. Mit der Leitinitiative Digitalisierung hat das Land Oberösterreich im Jahr 2016 ein 20-PunkteProgramm für die digitale Zukunft unseres Bundeslandes gestartet. Der Standort Oberösterreich soll damit zur "Digitalregion" werden. Im heurigen Jahr 2017 liegt der Schwerpunkt auf Themen wie Breitbandausbau, Informationssicherheit, generative Fertigung/3DDruck, Produktion, vernetztes Fahren sowie Fachkräfte. Die Umsetzung der Initiative wird von der oberösterreichischen Wirtschaftsagentur Business Upper Austria koordiniert.

\section{Digitalisierung ist in der Wirtschafts- und Forschungsstrategie verankert}

Ausgehend vom strategischen Ansatz, die Digitalisierung als Querschnittsmaterie zu betrachten, die in verschiedenste Bereiche hineinwirkt, folgt die Notwendigkeit, die Leitinitiative Digitalisierung auch kompatibel mit den wesentlichen strategischen Programmen und Initiativen auf regionaler, nationaler und europäischer Ebene zu gestalten. Allem voran steht dabei die Kohärenz mit dem Strategischen Wirtschafts- und Forschungsprogramm „Innovatives OÖ 2020". Dessen Leitlinien fokussieren auf die Kernkompetenzen Oberösterreichs und sind demnach auch für die Leitinitiative Digitalisierung relevant.

Als Querschnittsthema ist die Digitalisierung daher im Strategischen Wirtschafts- und Forschungsprogramm "Innovatives OÖ 2020" fest verankert. Ein wichtiger Schlüssel, um die Wettbewerbsfähigkeit weiterhin zu stärken, ist dabei die konsequente Vernetzung von Bildung, Forschung und Wirtschaft.
Aktuelles Beispiel dafür ist die Förderung von 14 innovativen Forschungsprojekten mit Schwerpunkt Digitalisierung. 27 Unternehmen - vom Leitbetrieb bis zum KMU - und praktisch alle Akteure des oberösterreichischen Innovationssystems - von der FH OÖ Forschungs \& Entwicklungs $\mathrm{GmbH}$, der Johannes Kepler Universität Linz, außeruniversitären Forschungseinrichtungen wie der RISC Software $\mathrm{GmbH}$, dem Software Competence Center Hagenberg GmbH und dem Research Center for Non Destructive Testing $\mathrm{GmbH}$ bis hin zur Forschungsleitgesellschaft des Landes OÖ, der Upper Austrian Research, sind beteiligt. 5,68 Millionen Euro werden vom Land Oberösterreich aus Mitteln der Forschungsförderung STAR dafür bereitgestellt. Inhaltlich geht es einerseits darum, Unternehmen mit geringerem Reifegrad den Einstieg in die Digitalisierung zu erleichtern - vorwiegend also um die technische Aufrüstung. Der zweite Schwerpunkt liegt bei Innovationsvorhaben, mit welchen die wertschöpfende Nutzung von Daten ausgebaut werden soll - etwa durch Predictive Maintenance und Predictive Analytics.

\section{Digitale Transformation aktiv unterstützen}

Oberösterreich hat mit international erfolgreichen Unternehmen und geballter Forschungskompetenz beim Thema Digitalisierung gute Chancen, um aus der digitalen Transformation gestärkt hervorzugehen. Der Vergleich mit angrenzenden europäischen Regionen zeigt aber auch deutlich die wirtschafts- und technologiepolitischen Handlungsfelder auf. Oberbayern beispielsweise ist bei Innovation, wissensintensiven Dienstleistungen und Technologie - etwa Breitbandverfügbarkeit oder der Nutzung neuer Technologien - teils deutlich weiter. Durch die traditionell starke Produktionswirtschaft gibt es in Oberösterreich zwar eine gute technologische Basis, gerade was z. B. Industrie 4.0 betrifft. Doch stehen viele Unternehmen vor dem Risiko, in einer international vernetzten Geschäftswelt den Anschluss zu verlieren. Gerade auf die vielen Unternehmen, die als Zulieferer erfolgreich sind, trifft das zu. Für sie ist die Gefahr groß, dass sie ihre Rolle in den künftigen Wertschöpfungsnetzwerken verlieren. Die Cluster der oberösterreichischen Wirtschaftsagentur Business Upper Austria unterstützen Unternehmen daher gezielt auf ihrem digitalen Weg. Cluster-übergreifende Initiativen - neben Connected Mobility auch Digital MedTech und Industrie 4.0/Produktion - vernetzen die IT-Welt mit anderen Branchen. Ziel ist es dabei stets, neue Geschäfts- und Beschäftigungsmodelle als auch Möglichkeiten, sich im internationalen Wettbewerb zu positionieren, anzustoßen. Aus diesen Kooperationen und Projekten heraus wurden bereits vielversprechende Instrumente entwickelt, um die Unternehmen fit für's Digital Business zu machen. Nachfolgend möchte ich einige dieser Initiativen exemplarisch vorstellen. Sie sollen die große Bandbreite an Themen und Instrumenten zeigen, mit denen in unserem Bundesland an der Digitalen Transformation gearbeitet wird.

Strugl, Michael, Landeshauptmann-Stv., Altstadt 17, 4021 Linz, Österreich (E-Mail: LHStv.Strug|@ooe.gv.at) 


\section{Analyse zeigt digitalen Reifegrad}

Eines der konkreten Unterstützungsangebote ist das "Reifegradmodell Industrie 4.0": Dabei handelt es sich um eine Art Navigationssystem, das firmenindividuell Richtung und Maßnahmen vorschlägt, um Unternehmen in kürzester Zeit "Industrie 4.0-fit" zu machen. Um im Wettbewerb weiter bestehen zu können, sind Advanced Manufacturing und die nachhaltige Produktionsoptimierung für jedes Unternehmen essentielle Bestandteile geworden. Zur Messung dieser Industrie 4.0-Reife wurde vom Mechatronik-Cluster gemeinsam mit dem Institut für Intelligente Produktion der FH-OÖ Campus Steyr das Reifegradmodell Industrie 4.0 entwickelt.

Bei diesem Modell wird anhand der Dimensionen Daten, Intelligenz und Digitale Transformation der Status quo eines Unternehmens in Bezug auf Industrie 4.0 gemessen. Ein strukturierter Prozess unterstützt dabei, Verbesserungspotenziale zu finden und diese zu realisieren. Neben operativen Unternehmensbereichen wie Produktion, Vertrieb oder Planung lassen sich mit dem Modell auch anstehende Projekte bzw. unternehmensstrategische Maßnahmen auf ihr Potenzial untersuchen und bewerten. Ein individueller Fahrplan zur Unternehmensoptimierung lässt sich daraus ableiten und erstellen. Im Fall des oberösterreichischen Sondermaschinenbauers Fill - wahrlich kein Anfänger in Sachen Digitalisierung und Automatisierung wurden beispielsweise bereits nach drei Tagen Optimierungspotenziale im Bereich der Konstruktion und Fertigung identifiziert.

Die Ergebnisse der Bewertungen fließen in eine BenchmarkDatenbank, wodurch sich aktuelle Marktsituationen in den Branchen identifizieren lassen. Ein anonymisierter Vergleich wird ermöglicht, der den eigenen Fortschritt jenem der Branche gegenüberstellt.

\section{Generative Fertigung beschleunigt die Entwicklung - bei geringeren Kosten}

Speziell im Bereich der Generativen Fertigung/3D-Druck (Additive Manufacturing) eröffnet die Mechatronik in Verbindung mit der zunehmenden Digitalisierung vollkommen neue Möglichkeiten. So unterstützt diese Technologie die Fertigung von Prototypen direkt aus CAD-Modellen, reduziert damit Produktentwicklungsschritte und Entwicklungskosten wesentlich und steigert somit die Effizienz in der Entwicklung.

Neben dem Prototypenbau sind es aktuell aber auch Anforderungen aus der Luftfahrt und dem Bereich Automotive sowie aus dem Werkzeugbau, die immer wieder neue Anwendungsfelder und Forschungsschwerpunkte für den Einsatz generativer Fertigung - egal ob mit 3D-Drucktechniken für Metalle oder mit Kunststoffen - liefern. Ziel ist es, dieses innovative Fertigungsverfahren in den oberösterreichischen Unternehmen breiter in die Anwendung zu bringen. Letztlich sollen aber auch neue Projektideen entstehen, denn Oberösterreich verfügt sowohl an der Johannes-Kepler-Uni als auch an der Fachhochschule OÖ über entsprechende Forschungsschwerpunkte.

\section{Vorausschauende Analytik/Produktionsdigitalisierung in der Kunststoffbranche}

In Kooperation mit F\&E-Einrichtungen und anderen Clustern setzt sich der Kunststoff-Cluster mit einer großen Herausforderung für seine Unternehmen auseinander: In der Kunststoffverarbeitung werden viele verschiedene "Rezepte" für Kunststoff eingesetzt, teilweise auch Recyclingmaterial. Da sich die Eigenschaften der eingesetzten Stoffe während der Produktion, aber auch noch während der Nutzung, ständig verändern, ist ihr "Verhalten" schwer vorauszusehen. Selbst aufwändige Simulationen bringen oft keine verwertbaren Ergebnisse. Um dennoch flexibel und effizient produzieren zu können, bietet die Digitalisierung durch vorausschauende Analytik große Chancen. Diese Potenziale sollen identifiziert und daraus
Projektideen zur Steigerung der Produktionsdigitalisierung in Spritzguss/Extrusion, der Lohnfertigung und dem Werkzeugbau abgeleitet werden.

\section{Künstliche Intelligenz: Oberösterreich auf dem Weg zur Kompetenzregion}

Neben dem Internet der Dinge, 3D-Druck und Virtual Reality ist es der Top-Technologietrend die kommenden Jahre: Künstliche Intelligenz - und da vor allem das Machine Learning. Dabei geht es um künstliche neuronale Netze, die es einer Maschine ermöglichen, aus Erfahrungen zu lernen und das Erlernte anzuwenden, z. B. Bilder zu erkennen.

Für den Standort Oberösterreich ist es essenziell, sich gezielt mit dem Thema Künstliche Intelligenz auseinanderzusetzen. Wir wollen aufbauend auf den bestehenden Stärken eine klare Strategie von der Grundlagenforschung über die angewandte Forschung bis hin zur Anwendung in den Unternehmen entwickeln. Ziel ist es, Oberösterreich zur international sichtbaren Kompetenzregion für KI zu machen. Dafür wird in den nächsten Monaten eine KI-Strategie für Oberösterreich mit konkreten Handlungsfeldern entwickelt. Es geht um die Frage, wo wir die nötigen Kompetenzen aufbauen können, um uns von anderen Regionen im Wettbewerb um Unternehmen, Fachkräfte und Investoren abzuheben. Ein wichtiger Puzzlestein ist die Ende Juni erfolgte Gründung des Al-Labs an der JohannesKepler-Universität Linz (JKU), mit dem ein Zentrum für Deep Learning entsteht. Deep Learning bezeichnet die Anwendung künstlicher neuronaler Netze mit vielen Schichten (daher "tief") auf die Lösung komplexer Aufgabenstellungen durch Lernen aus Beispieldaten, z. B. in Sprach- und Bilderkennung. Deep Learning wird erst durch neuartige Lernverfahren, große Datenmengen (Big Data) und die Verfügbarkeit hoher Rechenleistungen ermöglicht.

Die Erfindungen von Prof. Sepp Hochreiter, Vorstand des Instituts für Bioinformatik an der JKU, in der LSTM-Architektur (Long ShortTerm Memory) finden sich in praktisch jedem Smartphone und werden zur Spracherkennung, Texterkennung und Übersetzung eingesetzt. Die JKU und damit Oberösterreich hat beim Thema Deep Learning somit ein Alleinstellungsmerkmal, das durch den Aufbau des Al-Labs noch stärker sichtbar gemacht werden soll. Nicht nur, dass Prof. Hochreiter mit seinem Team dabei ist, weiter an Deep Learning zu forschen - das Al-Lab schafft auch die Möglichkeit zur verstärkten interdisziplinären Zusammenarbeit.

\section{Geballte Kompetenz in Oberösterreich}

Neben dem Zentrum für Deep Learning gibt es alleine an der JKU noch zahlreiche weitere Institute, die sich mit Teilaspekten der Künstlichen Intelligenz befassen. Dazu kommen die außeruniversitären Forschungseinrichtungen RISC Software, Linz Center of Mechatronics (LCM), das Software Competence Center Hagenberg ( $\mathrm{SCCH}$ ) und das K1-Zentrum prozfuture, die Fachhochschule OÖ mit dem Campus Hagenberg, das Ars Electronica Center in Linz und die bereits erwähnten Cluster der oberösterreichischen Wirtschaftsagentur Business Upper Austria: IT-Cluster, Mechatronik-Cluster, Automobil-Cluster und Medizintechnik-Cluster.

Gemeinsam mit innovativen Unternehmen aus der Industrie und Start-ups bildet dieses Netzwerk die solide Basis für eine notwendig Spezialisierung und Vertiefung des (Anwendungs-)Wissens um Künstliche Intelligenz. Letztlich geht es darum, mit all diesen Maßnahmen die Wettbewerbsfähigkeit und damit die Standortattraktivität Oberösterreichs zu verbessern.

Bis Ende des Jahres 2017 soll die KI-Strategie vorliegen. Den Auftakt für die Strategieentwicklung bildete ein Workshop in Hagenberg. Unter der Leitung von Dr. Burton Lee, Experte für Europäische 
Innovation und Entrepreneurship der Stanford Universität, diskutierten mehr als 70 Vertreter/innen aus der Forschung und aus Unternehmen über die (künftigen) Schwerpunkte in der Forschung und Anwendungen in der Wirtschaft.

\section{IT-Fachkräfte gefragt}

Sowohl auf Unternehmens- als auch auf Forschungsseite gilt: Im Innovations- und Standortwettbewerb ist die Verfügbarkeit von Fachkräften und deren Aus- und Weiterbildung der entscheidende Faktor, um die Chancen der Digitalisierung nutzen zu können. In den Unternehmen werden die Aufgaben immer komplexer, entsprechendes Know-how ist gefragt. Und es braucht auch exzellente Forscher/innen, die Innovationen vorantreiben. Es gilt daher, aktiv und vorausblickend auf die Herausforderungen zu reagieren. Mit der Leitinitiative Digitalisierung setzen wir entscheidende Schritte, um Oberösterreich für die digitale Zukunft fit zu machen. Ein besonderes Augenmerk liegt auf der beruflichen Ausund Weiterbildung. Denn digitale Kompetenz ist eine Schlüsselqualifikation, um die Wettbewerbsfähigkeit der Unternehmen zu sichern.

Oberösterreich kann sich mit seinen Ausbildungsstätten und möglichkeiten qualitativ auch im internationalen Vergleich sehen lassen. Der Engpass liegt aber in der Zahl der verfügbaren Fachkräfte. Von der HTL bis zur Universität und Fachhochschule gibt es ein breites Ausbildungsangebot: Die Fachhochschule OÖ bietet am Informatik-Campus Hagenberg sechs der Top10-IKT-Bachelorund -Masterlehrgänge an (Nr. 1 im FH-Ranking des Industriemagazins 2016). Insgesamt studieren 1.550 Student/innen in Hagenberg (z. B. Software Engineering, Energy Informatics, Information Security Management, Embedded Systems Design). Jährlich schlieBen 430 Absolvent/innen das Studium ab. Die Johannes-KeplerUniversität Linz zählt etwa 1.000 Studierende mit IKT-Schwerpunkt (z. B. Informatik, Informationselektronik, Wirtschaftsinformatik oder Computer Science). Und an den heimischen Höheren Technischen Lehranstalten (HTL) schließen jährlich knapp 400 Schüler/innen in einem der IKT-Schwerpunkt ab. Diese Zahl ist jedoch rückläufig

\section{Attraktivität von IT-Berufen und -Ausbildungen steigern}

Ein konkretes Projekt der Leitinitiative Digitalisierung hat daher zum Ziel, die Attraktivität von Ausbildungen im IKT-Sektor zu steigern Das gilt sowohl auf Uni- als auch auf HTL-Niveau. Dabei geht es darum, Schüler/innen für IKT-Ausbildungen in HTL und Maturanten/innen aus Gymnasien und berufsbildenden höheren Schulen für ein Studium im IKT-Bereich zu begeistern. Die dritte Zielgruppe sind internationale Studierende und Fachkräfte.

Ein weiteres Projekt ist der „Qualifizierungsverbund Digitale Kompetenz". Es beschäftigt sich mit Fachkräften in Produktionsunternehmen, die ihre Fähigkeiten um wichtige digitale Kompetenzen erweitern können. In einem Qualifizierungsverbund, der auch vom Arbeitsmarktservice Oberösterreich gefördert wird, schließen sich mehrere Unternehmen zusammen, um ihre Mitarbeiter gemeinsam bedarfsgerecht weiterzubilden.

Teil des Projektes ist auch eine „Kompetenzvorschau” zum Thema digitale Produktion. Dabei geht es um die Frage, welche Kompetenzanforderungen der digitale Wandel auch künftig an die Arbeitskräfte stellt und wie dafür frühzeitig zukunftsorientierte Lernangebote entwickelt werden können.

\section{Digitale Kompetenz als Erfolgsfaktor}

Eine aktuelle Studie ${ }^{1}$ im Auftrag der „Plattform für berufsbezogene Erwachsenenbildung" (PbEB) zeigt, dass digitale Kompetenz von den befragten Unternehmen als sehr wichtig eingeschätzt wird. Mehr als 60\% rechnen mit positiven Auswirkungen der Digitalisierung auf Arbeitseffizienz und Wettbewerbsfähigkeit. Zudem glauben knapp 60\%, dass die Qualität der Arbeitsleistung steigen und sich die Datensicherheit verbessern werde. Gleichzeitig sehen 90\% einen großen Bedarf an Weiterbildung im Bereich digitale Kompetenz.

In Oberösterreich werden Unternehmen auf ihrem Weg ins digitale Zeitalter aktiv unterstützt. Der neue „Qualifizierungsverbund Digitale Kompetenz" ist der erste Verbund österreichweit, der explizit digitale Aspekte in die berufliche Aus- und Weiterbildung bringt. Produzierende und produktionsnahe Unternehmen können ab sofort am Qualifizierungsverbund teilnehmen. Der Qualifizierungsverbund richtet sich vorwiegend an produzierende und produktionsnahe Unternehmen, die gezielt die digitale Kompetenz ihrer Beschäftigten erweitern wollen. Gemeinsam arbeiten das Land Oberösterreich, die oberösterreichischen Wirtschaftsagentur Business Upper Austria und das Arbeitsmarktservice Oberösterreich daran, ein dauerhaftes Netzwerk zur digitalen Kompetenzvermittlung zu etablieren. Das Land OÖ leistet zudem im Qualifizierungsverbund einen finanziellen Beitrag zur bestehenden Qualifizierungsförderung des Arbeitsmarktservice OÖ. Die Themen sprechen beinahe alle Unternehmensbereich an und sind sehr breit gefächert: Informationssicherheit, Datenschutzgrundverordnung der EU, Das $1 \times 1$ des digitalen Außenauftritts, Digitalisierung im Personalbereich, Reifegradmodell Industrie 4.0, Digitaler Stress, Bildbearbeitung, Kundenorientierte Produktion etc. Die genauen Inhalte der Schulungen können die Unternehmen mitbestimmen. Teil des Projektes ist auch eine „Kompetenzvorschau“ zum Thema digitale Geschäftsprozesse (z. B. Produktion, Vertrieb, Marketing, Organisation etc.), für deren Erstellung Unternehmen, Akteure der Arbeitsmarktpolitik und oberösterreichische Bildungsträger eng zusammenarbeiten werden. Dabei geht es um die Frage, welche Kompetenzanforderungen der digitale Wandel auch künftig an die Arbeitskräfte stellt und wie dafür frühzeitig zukunftsorientierte Lernangebote entwickelt werden können.

\section{Fachkräfte aus dem Ausland: Unterstützung durch Welcome2Upper Austria}

Unterstützung von Fachkräften aus dem Ausland bietet das Welcome2UpperAustria-Serviccenter. Es sorgt für eine Entlastung von Human-Ressource-Verantwortlichen durch die Bereitstellung umfangreicher, englischsprachiger Informationen und Serviceleistungen in zielgruppengerechter Aufbereitung. Die Website von Welcome2Upper Austria unterstützt die Recruiting-Maßnahmen von Unternehmen und bietet Internationals einen umfassenden Überblick zum Thema Leben und Arbeiten am Wirtschafts-, Arbeits- und Lebensstandort Oberösterreich.

\section{Wettbewerbsfähigkeit durch Digitalisierung zurückgewinnen}

Mit der Digitalisierung der Wirtschaft und der Gesellschaft werden die Karten im Standortwettbewerb gerade neu gemischt. Es gilt daher für unser Bundesland, diese neuen Chancen zu nutzen und zur Basis für die künftige Wettbewerbsfähigkeit Oberösterreichs zu machen. Strategische Projekte, konkrete Unterstützungsangebote und

${ }^{1}$ Für die Studie wurden von MAKAM Research Führungskräfte und Personalverantwortliche von 500 Firmen ab 20 Beschäftigten befragt (Studie "Weiterbildung 2017"; www.pbeb.at/presse; Stand: 08.06.2017) 
der große Schwerpunkt Qualifizierung - die Beispiele auf diesen Seiten geben einen Einblick in die aktuellen Aktivitäten des Wirtschaftsund Forschungsressorts des Landes OÖ. Der Schwerpunkt liegt dabei darauf, die Unternehmen auf ihren Weg zu begleiten und zu unterstützen. Die Anforderungen sind je nach Unternehmensgröße und digitalem Reifegrad höchst unterschiedlich. Ein wichtiger Erfolgsfaktor ist daher die maßgeschneiderte Unterstützung bis hin zur indivi- duellen Maßnahme auf Unternehmens- oder Branchenebene. Oberösterreich bietet diese Vielfalt an und hat so gute Chancen, aus der Digitalen Transformation stärker und wettbewerbsfähiger hervorzugehen.

Weitere Infos: www.digitalregion.at 